langrangian point $L_{1}$ both to be in continuous sunlight and to minimize orbital Doppler velocities relative to the Sun. The two helioseismological efforts are complementary, with GONG capable of probing differential rotation with depth and latitude, and SOI capable of studying more detailed dynamics of giant cells, zonal jets and smaller-scale flows closer to the surface. Several other helioseismological observations are also either under way or planned ${ }^{11}$. Inversion of the various oscillation data will be challenging, but from them should emerge a mapping of large-scale velocity and thermal structures throughout the convection zone.

Another promising route for examining flows near the surface involves high-resolution observation of granulation, tracking the movements of the cells as they evolve (see figure). Because the granules are convection cells confined to a shallow layer range near the surface, the plasma in which they reside should be swept along by the larger scales of motion that are deeper seated and more persistent. Such apparent advection of granules by flows of both mesogranular and supergranular scales is beautifully shown in the analysis ${ }^{12}$ of white-light images obtained with the SOUP instrument on Spacelab 2. Being closely packed and fairly uniformly distributed, the granules can serve as excellent flow tracers. However, granules typically have lifetimes of only about $10 \mathrm{~min}$, and thus tracking them requires frequent sampling of images and subtle pattern recognition algorithms. Further, provision must be made to filter out velocity and intensity contributions from the solar 5-min oscillations which would otherwise obscure the combined advection and evolution of granules. Analysis of the Spacelab 2 data $^{12}$ reveals the potential of using granules to track larger-scale flows, and other high-resolution solar observations from space are being planned.

Ground-based observations of solar granulation are severely impeded by blurring and image distortions resulting from turbulence in the Earth's atmosphere. Despite such difficulties, the impetus to study the smallest scales of turbulence visible on the Sun is great. In particular, the granular flows are energetic and capable of shuffling and twisting the magnetic fields in a manner that should lead to field reconnection and heating of the upper atmosphere. This notion is supported by time sequences of granulation images obtained using special telescopes when atmospheric seeing is at its best at sites such as Sacramento Peak and Pic du Midi, choosing only the sharpest images out of rapid bursts of photographs. Real-time image selection followed by digital processing to correct for distortions, as used by Brandt et al. ${ }^{1}$ at the Swedish telescope, point the way for future studies of solar granulation.

\title{
Fungi not fire damaged Aleppo Codex
}

THE Aleppo Codex, the earliest known manuscript of the Hebrew Pentateuch (the first five books of the Old Testament), was damaged by a fungus of the genus Aspergillus, rather than by fire as was previously assumed. The Codex was written on parchment in Tiberias in the tenth century. After a period in Jerusalem and Egypt, it was kept for 600 years in a synagogue in Aleppo (Halab), Syria. In 1947, the synagogue was set on fire

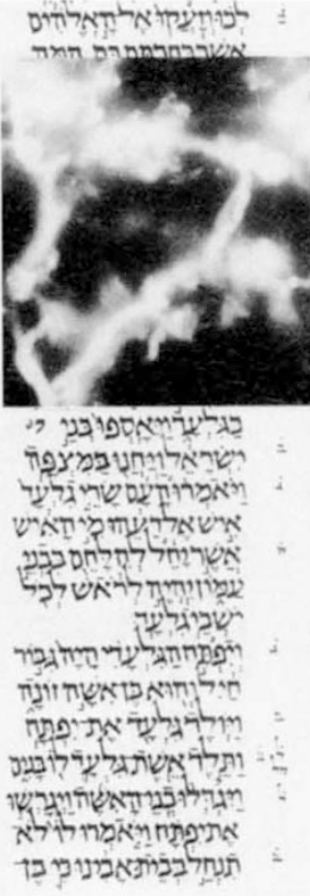

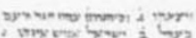
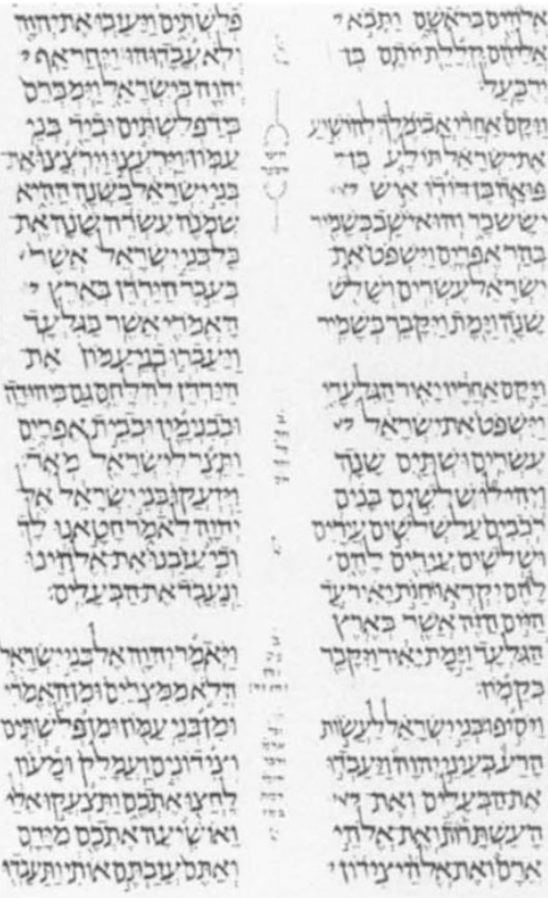
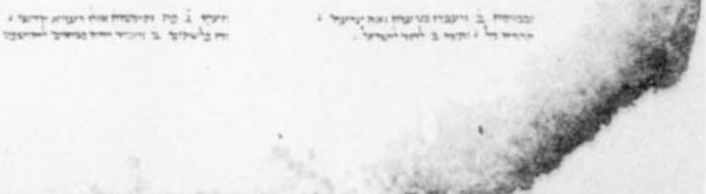

A page from the Codex showing typical discolouration and deterioration in the outer lower corner. Inset: fluorescence photomicrograph of fungus-like filaments in the damaged areas. Preparations mounted in the fluorescent fungal stain Cellufluor (Polysciences), $\mathrm{KOH}$ and glycerol. $\times 600$.

but 295 pages, about two thirds of the original manuscript, survived and were given on permanent loan by the Jewish community in Aleppo to the Israel Museum for restoration and exhibition. The fate of the missing pages is unknown ${ }^{1,2}$.

The outer lower corners of all the pages are aubergine-coloured and brittle (main figure) and the parchment is weakened in these areas, which were subject to constant handling by people turning the pages. This pattern is more characteristic of damage by fungi than by fire ${ }^{3}$ and, early signs of deterioration were already visible in $1910,{ }^{4}$. Itzhack Polacheck and colleagues* have now examined fragments from the damaged areas with phase-contrast and fluorescence microscopy, which reveals positive fluorescent septate filaments (inset) not seen in fragments from the undamaged areas. Specific fluorescent-antibody staining identifies the filaments as Aspergillus hyphae.

1. Shamosh, A.Ha-Keter. The Story of the Aleppo Codex (Yad Ben-Zvi/Hebrew University, Jerusalem 1987).

2. Goshen-Gottstein, M.H. Textus I, 17-58 (1960)

3. Reed, R. Ancient Skins, Parchments and Leathers (Seminar, London, 1972).

4. Segall, J. Travels through Northern Syria (London Soc. Promoting Christianity among Jews, London, 1910).

1. Brandt, P.N., Scharmer, G. B Ferguson, S , Shine, R.A Tarbell, T.D. \& Title, A.M. Nature 335, 238-240 (1988).

2. Scharmer, G. in The Role of Fine-Scale Magnetic Fields in the Structure of the Solar Atmosphere (ed. Vázquez, M 349-353 (Cambridge University Press, 1987)

3. Zahn, J.-P. in Solar and Stellar Physics, Lecture Notes in Physics Vol. 292 (eds Schrötet, E.H. \& Schüssler, M.) 55 71 (Springer, New York, 1987).

4. Hurlburt, N.E., Toomre, J. \& Massaguer, J.M. Astrophys. J. 311, 563-577 (1986)

. Nordlund, A. \& Stein, R.F. Astrophys. J. (in the press)

6. Gilman, P.A. \& Miller, J. Astrophys. J. 61, 585-608 (1986)

. Glatzmaier, G.A. in The Internal Solar Angular Velocity
* I. Polacheck (Department of Clinical Microbiology, The Hebrew University-Hadassah Medical Center, Jerusalem 91120, Israel); I.F. Salkin (New York State Department of Health, Albany, New York 12201); D. Schenhav, L. Ofe and M. Maggen (Israel Museum, Jerusalem); J.H. Haines (New York State Museum, Albany, New York 12230, USA) (eds Durney, B.R. \& Sofia, S.) 263-274 (Reidel, Dordrecht, 1987)

8. Hart, J.E., Glatzmaier, G.A. \& Toomre, J. J. Fluid Mech. 173, 519-544 (1986)

9. Gough, D.O. \& Toomre, J. Solar Phys. 82, 401-410 (1983).

10. Harvey, J.W., Kennedy, J.R. \& Leibacher, J.W. Sky \& Telescope 74, 470-476 (1987).

1. Libbrecht, K.G. Space Sci. Rev. (in the press)

12. Simon, G.W et al. Astrophys. J, 327, 964-967 (1988).

Juri Toomre is in the Joint Institute for Laboratory Astrophysics, University of Colorado, Boulder, Colorado 80309, USA 